\title{
Leadership Perception during COVID-19 Pandemic: The Critical Criticism on Surgical Leadership
}

\author{
Adeel Abbas Dhahri ${ }^{1 *}$, Ubaid Ur Rehman ${ }^{2}$, Syeda Shahana Ali ${ }^{3}$, Muhammad \\ Hussnain Iqbal ${ }^{4}$, Alexandra Lupu ${ }^{5}$, Mehvish Adeel Dhahri ${ }^{6}$, Jonathan S. Refson ${ }^{5}$ \\ ${ }^{1}$ Department of Surgery, Royal Infirmary Hospital Edinburgh, Scotland, United Kingdom. \\ ${ }^{2}$ Norfolk and Norwich University Hospital, United Kingdom. \\ ${ }^{3}$ Dumfries and Galloway Royal Infirmary, Scotland, United Kingdom. \\ ${ }^{4}$ Royal London Hospital, London, United Kingdom. \\ ${ }^{5}$ The Princess Alexandra Hospital NHS Trust, Harlow, United Kingdom. \\ ${ }^{6}$ Self-employed, Edinburgh, Scotland, United Kingdom.
}

\begin{abstract}
Coronavirus disease 2019 (COVID-19) has altered the expectations of team leaders by team members while they seek clear and efficient decisions. We aim to assess the perception of leadership by the surgical team members and their perception of the most important quality of leadership. We conducted an online national cross-sectional survey among the surgical doctors working in the National Health Service United Kingdom, between $15^{\text {th }}$ July 2020 till $31^{\text {st }}$ August 2020. The questionnaire, based on rating scale items, focused on effects of changes in work-pattern, support and communication at workplace, the status of leadership and understanding of different leadership attributes. Out of two hundred and thirteen participants, majority of respondents were middle-grade registrars $(n=92,43.7 \%)$. Most of the doctors $(147,81.7 \%)$ were never re-deployed to the high-risk areas. 85(39.9\%) expressed that COVID-19 brought distress affecting their morale. 106(49.8\%) thought that arrangement of Personal Protective Equipment (PPE) was the responsibility of the Head of Department. The majority, of those who fell ill, felt adequately supported by their seniors. Most participants identified departmental leadership as satisfactory $(n=126,59.2 \%)$. There was a mixed response to the importance of leadership attributes defined by the Faculty of Medical Leadership and Management. Stress at the workplace with reduced morale, during a crisis like COVID-19 pandemic, can significantly compromise leadership perception among team members. Understanding of basics of leadership by the team members can improve departmental efficiency and as a result improve patient care.
\end{abstract}

Keywords: Surgical Leadership; Surgical Teams; Perception; COVID-19.

\section{Introduction}

Hospital management is a combination of professional leaders and functional members of the team with a range of motivation and engagements of goals. Due to differences in agendas at a time, there can often be leading to resultant abrasions of stereotypical distractions between team members and leaders [1]. These abrasions can lead to an influence on leadership perception by values depending upon the state of affairs and challenging events [2].

* Corresponding author: adeeldhahri@hotmail.com

doi) http://dx.doi.org/10.28991/SciMedJ-2021-0303-6

$>$ This is an open access article under the CC-BY license (https://creativecommons.org/licenses/by/4.0/).

(C) Authors retain all copyrights. 
Different frameworks and standards are available to address leadership in the hospital environment and beyond. The Faculty of Medical Leadership and Management (FMLM) updated the standards focusing on leadership and management in 2016, specifically for medical professionals. A good leadership not only brings better productivity within the organisation by lifting the spirit but also resultant improved patient outcome [3]. These 'Leadership and Management Standards for Medical Professionals' focus on four primary standards like self-awareness and development, effective team-player, corporate responsibility and system leadership. These standards within an organisation aspire leadership driving motivation and energy within a team, with resultant collaborative and effective team-work. As a responsible leader in a team, strategic and operational delivery boosts positive practice in the healthcare environment for better patient outcome [4].

Coronavirus disease 2019 (COVID-19) pandemic has restructured the policies within the healthcare departments, to adapt to an extensive global crisis. Such a crisis may bring frustrations among the team members when they are lost in emotions and burn-out added-up by substandard inter-departmental communication, with decisive poor perception of the departmental leadership. Flexibility and transformation remain analytical for unique as well as successful leadership. Since this inevitable pandemic, both leaders and followers, within the team, struggle with their responsibilities as they treat patients whilst protecting themselves [5-8].

We assumed that COVID-19 pandemic had brought the obscured perception of surgical leadership among team members. We conducted this study to assess the perception of leadership by the members of the surgical department and their perception of the essential qualities within leadership based on FMLM.

\section{Methods and Materials}

This multi-centre cross-sectional study focused on surgical doctors working at the National Health Service (NHS) United Kingdom, and was conducted between $15^{\text {th }}$ July 2020 till $31^{\text {st }}$ August 2020. An anonymous online questionnaire, using Google Survey, was distributed across the United Kingdom (UK), of all grades through email and social media platforms, including WhatsApp. Participants invited to complete the survey were of junior doctors including Foundation Year level one (FY1) and level two (FY2)/ Senior House Officer (SHO), middle grades (ST3+ - Specialty Trainee equivalent) and consultants (who were not active departmental leads).

The questionnaire containing thirteen (13) close-ended questions, based on a rating scale, discussed individual's perception about their opinions regarding their departmental leadership. Additionally, questions focused on changes in work-pattern, the effect of work pattern on mental status, support by seniors and colleagues, level of communication, and understanding of different leadership perspectives. With reference to the Faculty of Medical Leadership and Management (FMLM) defined Leadership and Management Standards for Medical Professional, participants were also asked to categorise and prioritize competencies as they see essential at their work-place. Terms used to define categories of FMLM were described to the participants as; attentiveness for self-awareness, chair for corporate responsibility, synergy for team-work, and vision for system leadership.

Statistical analyses were performed using SPSS 21.0. All the participants were informed about the objective of the study. Ethical approval was not required as this non-interventional study was based on doctors who voluntarily participated in the anonymous online survey. The study has been reported in line with STROBE guidelines [9].

\section{Results}

A total of 213 participants voluntarily responded to this national online survey. All the participants were not actively involved in any leadership role to avoid bias.

\subsection{Demographics}

Majority of the respondents were middle grade registrars $(n=92,43.7 \%)$, followed by FY2/SHO $(n=80,37.6 \%)$, consultants $(\mathrm{n}=25,11.7 \%)$ and FY1's $(\mathrm{n}=15,7 \%)$.

\subsection{Leadership Perspectives}

The participants were asked to state about the level of leadership they have in their department from very good to bad. 40(18.8\%) responded as very well, 86(40.4\%) as good, 62(29.1\%) as neutral while 25(11.7\%) as bad (Figure 1).

Participants in the questionnaire were also asked to rank each quality of leadership according to the framework (Attentiveness, Chair, Synergy and Vision). Through all stages of doctors from FY1 to consultants, various characteristics were preferential rather than any specific characteristic. However, on average chair being selected as the most valuable while synergy was consistently least important (Figure 2). 


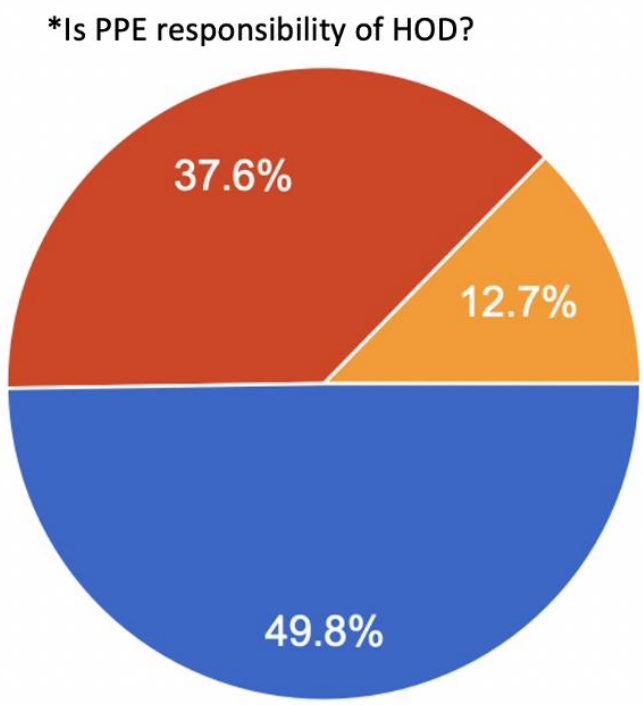

Yes

No

No comment

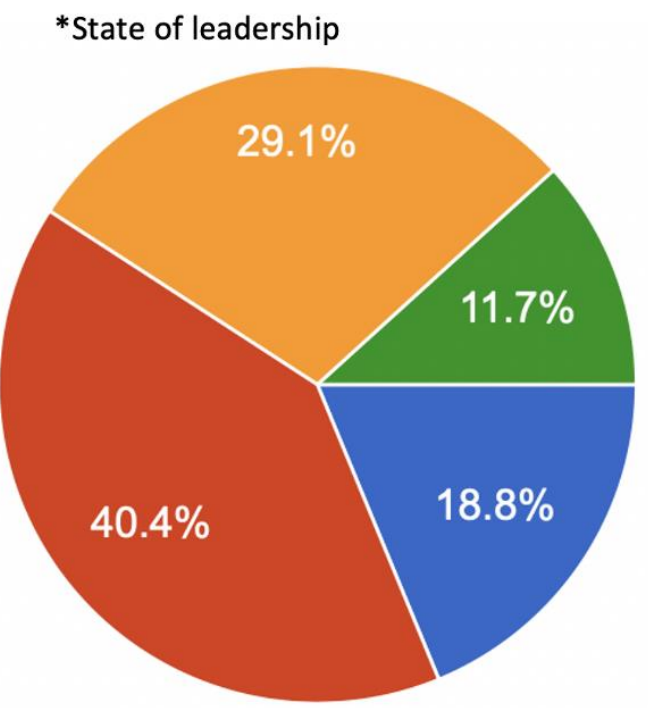

Very good

Good

Neutral

Bad

Figure 1. Perception of departmental leadership $(n=213$; PPE = Personal Protective Equipment; HOD = Head of Department)

\section{What is most important for you at work?} ( 1 = most important, 5 = least important)

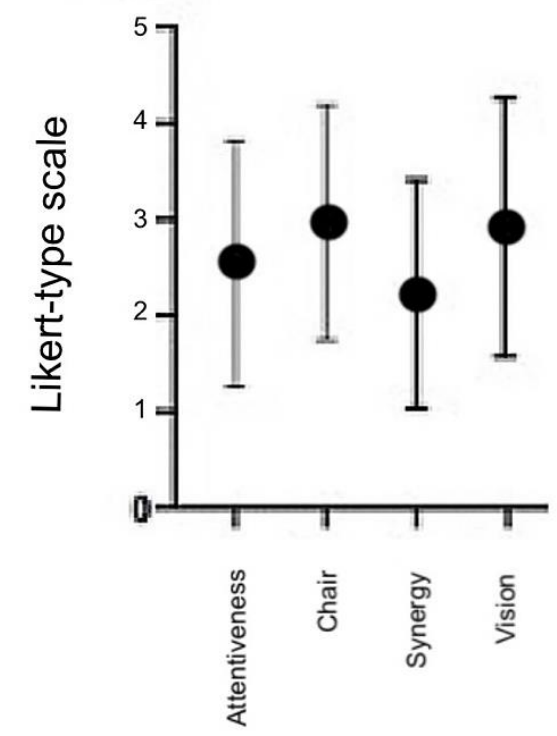

Figure 2. Teams' preference of leadership attributes

\subsection{Changes in Work-pattern}

A majority $(n=158,74.2 \%)$ of the doctors felt that the focus of healthcare shifted towards public health from patientcentred.

A good sizeable participant $(n=147,69 \%)$ agreed that their rota changed due to the pandemic, and $172(81 \%)$ did not experience any redeployment. Among Medical \& Surgical specialities $(n=180), 147(81.7 \%)$ were never redeployed out of their department to the high-risk areas, while 11(6.1\%) were redeployed once and 22(12.2\%) were redeployed more than once. Among those who were redeployed $(n=33), 8(24.2 \%)$ felt that they were not adequately trained before to redeployment, $16(48.5 \%)$ were partially trained, and $9(27.3 \%)$ were adequately trained to work with confidence in highrisk areas (Table 1). 
Table 1. Experience of redeployment to high-risk areas

\begin{tabular}{|c|c|}
\hline & $\mathrm{n}=180$ \\
\hline 1. Redeployed to high-risk areas & $33,18.33 \%$ \\
\hline - Once & $11,6.1 \%$ \\
\hline - More than once & $22,12.2$ \\
\hline 2. Adequately trained & $9,27.3 \%$ \\
\hline 3. Partially trained & $16,48.5 \%$ \\
\hline 4. Not adequately trained & $8,24.2 \%$ \\
\hline
\end{tabular}

\subsection{Effect of Work Pattern on Mental Status}

There was a very balanced view of distress and morale being affected by the change in work pattern. 83(39\%) stated that that the COVID-19 related workplace situation did not bring distress to them to affect their morale while another $85(39.9 \%)$ expressed reduced morale with distress and 45(21.1\%) were not sure about it (Figure 3 ).
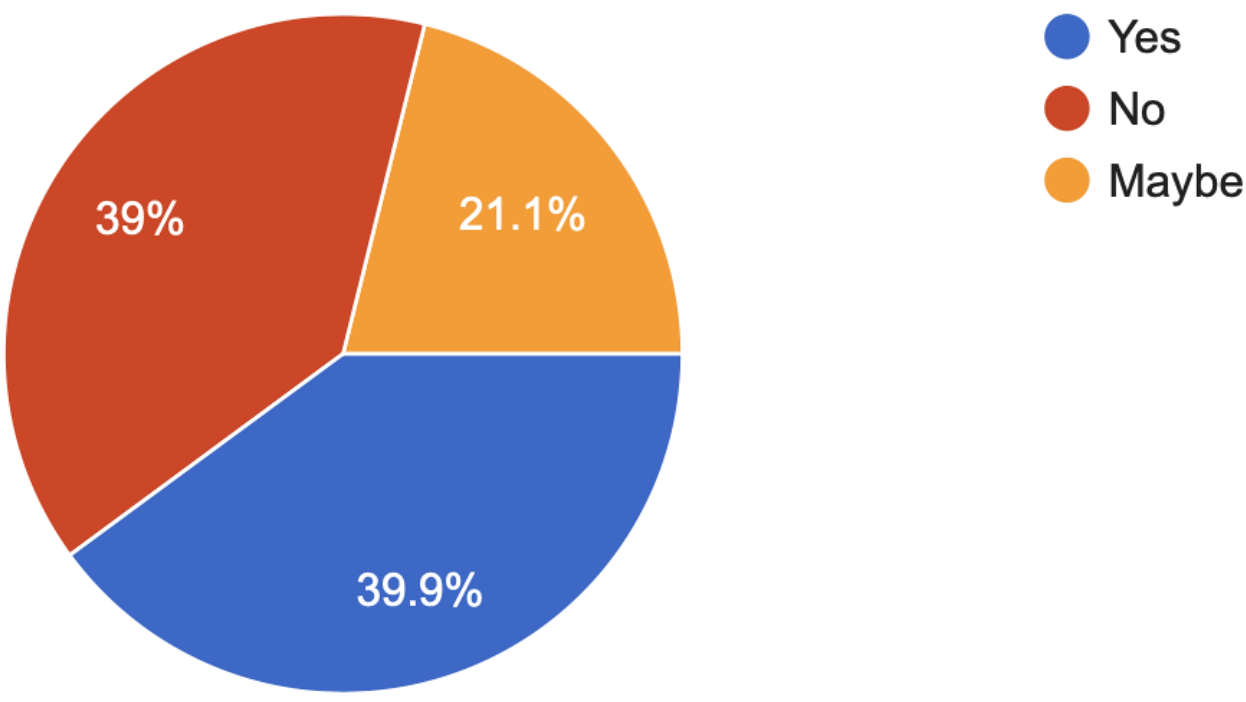

Figure 3. Work pattern leading to distress and reduced morale $(n=213)$

\subsection{Support by Seniors}

Among 213, 106(49.8\%) thought that arrangement of Personal Protective Equipment (PPE) was the responsibility of the Head of Department while $80(37.6 \%$ ) thought otherwise (Figure 1). 96(45.1\%) had to take time off due to illness during the pandemic and, out of these, $75(78 \%)$ of these felt adequately supported by their seniors, during this pandemic.

\subsection{Communication}

As during any crisis, communication was a vital part of the COVID-19 pandemic. $90(42.3 \%)$ of the doctors thought that the level of communication, within the department, improved during the pandemic while 54(25.4\%) felt it got worse than pre-COVID (Table 2).

Table 2. Communication between team-members during COVID-19

\begin{tabular}{ll}
\hline & $\mathrm{n}=213$ \\
\hline Improved & $90,42.3 \%$ \\
\hline Worse & $54,25.4 \%$ \\
\hline Same as pre-COVID & $69,32.3 \%$ \\
\hline
\end{tabular}




\section{Discussion}

"If I had to reduce the responsibilities of a good follower to a single rule, it would be to speak truth to power." Warren Bennis.

The focus of this cross-sectional study was to understand the perception of leadership and management within hospitals by its team members during a period of crisis.

Stress, as the psychological consequence of COVID-19 pandemic, is the main confounding factors at the workplace. World Health Organisation (WHO) has already recognised the psychological effects of the current pandemic [10, 11]. It can arise from multiple reasons like changes in work-pattern, after falling ill, feeling of being unsupported by the seniors, poor departmental communication and higher than average expectations from the departmental leadership. Although doctors are not expected to work outside their competences, the current pandemic has shocked the health system as hospitals reshape their working and service pattern to overcome demand beyond capacity in high-risk areas. Such reshaping can be related to physical and psychological burn-out. Resilience may be expected with quick changes in the system on a regular and more frequent basis, it is the responsibility of the leadership within the hospitals at all levels, to support junior doctors by training them before redeployment to high-risk areas. During COVID-19 pandemic, literature found willingness to work with dedication among junior doctors $[6,12]$. Our survey confirmed that at the majority of workplaces did have their work-pattern did change with the focus of healthcare shifting towards public health. However, in our study, only a few were redeployed to the high-risk area with two-third of them felt adequately trained before redeployment.

Changes in work-pattern are never without the expense of psychological and physical effects with team members aspiring to visible and transparent leadership to avoid stress and reduced morale. Also, in situations where work pressure can trigger the burn-out among team members, expectations can aggravate and complicate tasks for leaders. Despite, both leadership and followership being two-sides of the coin, the process of commitment, vision, and performance start with leadership. Good communication can be a crucial factor during such period to reassure team on board, all queries and worries reasonably answered [13, 14]. Maintaining a high level of comprehensive communication between all team members helps to relay information quickly but also with regular feedbacks from team [8]. Our survey demonstrated, $50 \%$ of the doctors recognised improvement in the communication between their teams during current pandemic, and the majority also felt supported and satisfied with the departmental leadership.

As the world witnessed mortality among healthcare workers, meticulous use of PPE was made mandatory to minimise the risk of COVID-19 transmission, especially during aerosol-generating procedures. Protocols and guidelines were introduced at all levels, including by Public Health England [11, 15]. It is the responsibility of the head and managers of the hospitals to provide standard PPE for the well-being of the healthcare workers in the hospital. However, departmental leaders are required to act as a bridge to convey team-members concerns, about the adequacy of availability of PPE, to the stake-holders [8]. Our results demonstrated that nearly half of the responders perceived it was departmental lead responsibility to arrange PPE for them. This perception reflects the higher expectations amongst surgical team members during the crisis.

With higher expectations, while team-members felt safer with their departmental leadership, surgical doctors ranked the leadership standard as they perceived. Most of the surgical doctors in our survey felt that corporate responsibility was the most critical aspect of the leadership for better departmental performance and clinical outcome. Since 2011, FMLM has been an active professional body working to improve medical leadership standards. Leadership and Management Standards for Medical Professional was first launched in 2015 and has been updated once since then. It has four domains which revolve around Self-awareness and Self-development to improve resilience and drive, Team play for effective team-work and collaboration, Corporate responsibility for changing the culture to improve and innovate. The last domain is System leadership, which is focused on translating effective organisational policy for better team and clinical outcome. FMLM leadership and management standards have encouraged the idea of leadership as a prime responsibility of all doctors at all stages of their professional career. Ensuring patient safety while systematically promoting safe culture at a workplace for the patient is key $[3,16]$. In a healthcare environment, recognition of leadership can be strenuous ergonomics of effective working pattern. In such a workplace, where perceptions vary about quality and quantity, it becomes challenging to execute purposeful and unbiased goal [17]. In our study, there were specific attributes in the leadership that were more important to the staff in COVID times. Attentiveness and synergy suggested that the staff wanted their leader to pay attention to their concerns and work with them. Simultaneously, they wanted a leader to have a vision and give them hope in difficult times. Although most of our participants perceived corporate responsibility as the supreme quality of the leadership, there was a mixed response to the importance of leadership attributes defined by the FMLM (Figure 2).

We identified, through our survey, that team members need to trust their leadership when leaders take actions at the broader aspect to prepare and plan for unseen events during a time of acute crisis. Understanding of quality improvement and all the domains of FMLM in the team is crucial and has a direct impact on the workplace and clinical outcome. 


\section{Conclusion}

Leadership during a crisis is demanding and stressful. On the other hand, followers in the team may often act as a mirror image of the leaders, to provide criticism and feedback. Positive criticism on the leaders can help effective planning for organisational governance and better patient outcome. Stress and reduced morale among followers can significantly compromise leadership perception among them. Safe-guarding and well-being of the followers by the team leaders is directly related to the perception of followership. While COVID-19 tests leadership perception, understanding the basics of leadership by the team members is precious for a better workplace apprehension and thus the organisational outcome.

\section{Declarations}

\subsection{Author Contributions}

Conceptualization, A.A.D.; data collection, A.A.D., U.R., S.S.A., A.L. and M.A.D.; data analysis and interpretation, A.A.D., M.H.I., A.L. and M.A.D.; writing — original draft preparation, A.A.D., U.R., S.S.A., M.H.I., M.A.D. and J.R.; writing-review and editing, A.A.D., M.H.I. and J.R. All authors have read and agreed to the published version of the manuscript.

\subsection{Funding}

The authors received no financial support for the research, authorship, and/or publication of this article.

\subsection{Ethical Approval}

Ethical approval was not required as this non-interventional study was based on doctors who voluntarily participated in the anonymous online survey.

\subsection{Data Availability Statement}

The data presented in this study are available in article.

\subsection{Conflict of Interest}

The authors declare that they have no known competing financial interests or personal relationships that could have appeared to influence the work reported in this paper.

\section{References}

[1] Nigam, A. (2018). Multiple and competing goals in organisations: insights for medical leaders. BMJ Leader, 2(3), 85-86. doi:10.1136/leader-2018-000112.

[2] Asamani, J. A., Naab, F., Ofei, A. M. A., \& Addo, R. (2016). Do leadership styles influence productivity? British Journal of Healthcare Management, 22(2), 83-91. doi:10.12968/bjhc.2016.22.2.83.

[3] Kyratsis, Y., Armit, K., Zyada, A., \& Lees, P. (2016). Medical leadership and management in the United Kingdom. Australasian Psychiatry, 24(3), 240-242. doi:10.1177/1039856216635909.

[4] Lees, P. (2015). Professionalising medical leadership. Future Hospital Journal, 2(3), 208-210. doi:10.7861/futurehosp.2-3-208.

[5] Iserson, K. (2020). Healthcare Ethics during a Pandemic. Western Journal of Emergency Medicine, 21(3), 477-483. doi:10.5811/westjem.2020.4.47549.

[6] Brethauer, S. A., Poulose, B. K., Needleman, B. J., Sims, C., Arnold, M., Washburn, K., ... Pawlik, T. M. (2020). Redesigning a Department of Surgery during the COVID-19 Pandemic. Journal of Gastrointestinal Surgery, 24(8), $1852-1859$. doi:10.1007/s11605-020-04608-4.

[7] Linton, J., \& Farrell, M. J. (2009). Nurses' perceptions of leadership in an adult intensive care unit: A phenomenology study. Intensive and Critical Care Nursing, 25(2), 64-71. doi:10.1016/j.iccn.2008.11.003.

[8] Dhahri AA, Iqbal MR, Darwish N., \& Vijay V. (2020). The Importance of Followership in a Crisis- Lessons Learnt From a Survey of Junior Doctors' Perception of Leadership during the COVID-19 Pandemic. Journal of Universal Surgery 8 (6):4

[9] Elm, E. von, Altman, D. G., Egger, M., Pocock, S. J., Gøtzsche, P. C., \& Vandenbroucke, J. P. (2007). Strengthening the reporting of observational studies in epidemiology (STROBE) statement: guidelines for reporting observational studies. BMJ, 335(7624), 806-808. doi:10.1136/bmj.39335.541782.ad.

[10] World Health Organization. Mental health and psychosocial considerations during the COVID-19 outbreak. Available online: https://www.who.int/docs/default-source/coronaviruse/mental-health-considerations.pdf (accessed on 6 September 2020). 
[11] Kursumovic, E., Lennane, S., \& Cook, T. M. (2020). Deaths in healthcare workers due to COVID-19: the need for robust data and analysis. Anaesthesia, 75(8), 989 - 992. doi:10.1111/anae.15116.

[12] Rimmer, A. (2020). Covid-19: what do trainees need to know? BMJ, m1276. doi:10.1136/bmj.m1276.

[13] Shanafelt, T., Ripp, J., \& Trockel, M. (2020). Understanding and Addressing Sources of Anxiety among Health Care Professionals during the COVID-19 Pandemic. JAMA, 323(21), 2133. doi:10.1001/jama.2020.5893.

[14] Sohmen, V. S. (2013). Leadership and teamwork: Two sides of the same coin. Journal of IT and Economic Development, 4(2), $1-18$.

[15] Public Health England (2020) COVID-19 personal protective equipment (PPE). Available online: https://www.gov.uk/ government/publications/wuhan-novel-coronavirus-infection-prevention-and-control/covid-19-personal-protective-equipmentppe (accessed on 11 Sep. 2020).

[16] Leadership and management standards for medical professionals. Faculty of Medical Leadership and Management. Available online: https://www.fmlm.ac.uk/standards (accessed on 15 July 2020).

[17] Otara, A. (2011). Perception: A Guide for Managers and Leaders. Journal of Management and Strategy, 2(3), 21-24. doi:10.5430/jms.v2n3p21. 